\title{
Desempenho reprodutivo pós-parto de vacas de corte submetidas a indução/sincronização de cio ${ }^{1}$
}

\section{Cássio Cassal Brauner ${ }^{2}$, Marcelo Alves Pimentel ${ }^{3}$, Jaqueline Schneider Lemes ${ }^{4}$, Cláudio Alves Pimentel ${ }^{5}$, José Carlos Ferrugem Moraes ${ }^{6}$}

\author{
${ }^{1}$ Pesquisa financiada pelo CNPq, PPGZ - FAEM - UFPel. \\ 2 FAEM - UFPEL, UFPEL/FAEM/DZ, Campus Universitário s/n, cx. postal 354, Pelotas, RS, CEP: 96010970. \\ ${ }^{3}$ Departamento de Zootecnia , FAEM - UFPEL. \\ ${ }^{4}$ Mestranda - PPGZ - FAEM/UFPel. Bolsista do CNPq. \\ ${ }^{5}$ Pesquisador CNPq. \\ ${ }^{6}$ Embrapa Pecuária Sul.
}

RESUMO - Para avaliar o desempenho reprodutivo de vacas de corte submetidas à indução/sincronização de cio, foram utilizadas 42 vacas da raça Aberdeen Angus com condição corporal 3 (escala de 1-5) sob manejo extensivo. A produção de leite foi estimada pelo método pesagem-mamada-pesagem. Nos exames ginecológicos, utilizou-se aparelho de ultrasonografia e palpação trans-retal. Foram considerados fatores fixos nível de produção de leite (acima e abaixo da média de produção das vacas), gestação, condição reprodutiva pré-acasalamento (CRPA), anestro superficial (tônus uterino, folículos $\geq 10 \mathrm{~mm}$ e ausência de CL) e anestro profundo (ausência de tônus uterino, folículos < 10 mm e ausência de CL) e a resposta à indução/sincronização de cio (RISC). As variáveis analisadas foram os pesos pré-parto, ao parto, ao acasalamento e à concepção, o peso à desmama, o ganho médio de peso diário do parto ao desmame e a produção de leite total. A condição reprodutiva pré-acasalamento foi influenciada pelos pesos pré-parto e ao parto, comprovando que o melhor desempenho pré-parto e ao parto favorece o restabelecimento da atividade reprodutiva em vacas com condição corporal (CC) moderada no período pós-parto. A classificação da condição reprodutiva pré-acasalamento em vacas de corte pode ser um recurso para determinar o desempenho reprodutivo de vacas de corte submetidas à indução/sincronização de cio, uma vez que vacas em anestro superficial tendem a apresentar melhor resposta ao protocolo. Em vacas de corte com condição corporal moderada, o desempenho ponderal pré-parto influencia a condição reprodutiva pré-acasalamento, aumentando a probabilidade de melhor desempenho reprodutivo.

Palavras-chave: anestro, produção de leite, reprodução

\section{Postpartum reproductive performance of beef cows in moderate body condition submitted to estrus induction/synchronization}

\footnotetext{
ABSTRACT - To assess the reproductive performance of beef cows submitted to estrus induction/synchronization, 42 Aberdeen Angus cows with body condition 3 (1-5 scale) under extensive management were used. The milk production was estimated through the weight-suckling-weight method. For gynecological examinations, ultrasound equipment and trans-rectal palpation were used. The milk production level (above and below the average production of cows), pregnancy, prebreeding reproductive condition (CRPA), surface anoestrus (uterine tone, follicles $\geq 10 \mathrm{~mm}$ and absence of CL) and deep anoestrus (no uterine tone, follicles $<10 \mathrm{~mm}$ and absence of $\mathrm{CL}$ ) and the estrus induction/synchronization response (RISC) were considered as constant factors. The variables analyzed were the pre-delivery, delivery weight, mating and conception weights, weaning weight, the average daily weight gain from birth to weaning and total milk production. The prebreeding reproductive condition influenced the pre-birth and birth weights, showing that the better pre-birth and birth performance favors the restoration of reproductive activity in cows with moderate body condition (CC) in the post-partum period. The classification of the pre-breeding reproductive condition in beef cows can be a resource to determine the reproductive performance of beef cows submitted to estrus induction/synchronization, since cows in surface anoestrus tend to have better response to the protocol. In beef cows with moderate body condition, the pre-birth weight performance affects the prebreeding reproductive condition, increasing the likelihood of better reproductive performance.
}

Key Words: anoestrus, milk production, reproduction 


\section{Introdução}

Anestro é o fator de maior impacto negativo sobre a fertilidade pós-parto, pois aumenta o intervalo do parto ao primeiro cio, reduzindo a produção de bezerros e causando perdas econômicas na produção de bovinos de corte. É um período de transição da aciclicidade para a ciclicidade, no qual o eixo hipotalâmico-hipofisiárioovariano-uterino se recupera de uma gestação. Diversos fatores influenciam a duração do período de anestro pósparto: os de maior impacto são a nutrição e amamentação (Short et al., 1990; Williams, 1990; Hess et al., 2005).

Com a finalidade de antecipar o retorno à ciclicidade, aumentar a eficiência de sincronização de cio e a utilização da inseminação artificial (IA), métodos para induzir o retorno à ciclicidade podem ser utilizados após 30-40 dias pós-parto em animais em condição corporal moderada ou superior.

A utilização de progesterona exógena e seus análogos é um dos métodos utilizados para mimetizar um ciclo estral curto e aumentar a freqüência pulsátil de LH (Yavas \& Walton, 2000; Jaume \& Moraes, 2002; Bastos et al., 2004; Day, 2004). A utilização de estradiol associado a implantes de progesterona em protocolos de indução/sincronização de cio objetiva suprimir um folículo dominante e estabelecer a emergência de uma nova onda de crescimento folicular em três ou quatro dias, independentemente do estágio de desenvolvimento do folículo dominante no início do tratamento (Bó et al., 1995; Burke et al., 2003).

A produção de leite em vacas de corte é determinada pela capacidade de alimentação do bezerro (Pimentel et al., 2006). A lactação é um estado homeorético (dominante) no qual ocorrem adaptações no metabolismo, em tecidos e órgãos da vaca, sempre priorizando a síntese de leite (Bauman \& Currie, 1980). Diversos estudos demonstram efeito negativo da lactação e da presença do bezerro sobre o desempenho reprodutivo pós-parto de vacas de corte (Short et al., 1990; Williams et al., 1990). No entanto, Pimentel et al. (2005), estudando a lactação de vacas de corte criadas extensivamente no Rio Grande do Sul, demonstraram que a produção de leite não é um dos principais fatores limitantes sobre a fertilidade pós-parto. Neste estudo, objetivou-se determinar a influência do desempenho pré e pós-parto e da produção de leite sobre os desempenhos produtivo e reprodutivo de vacas de corte em condição corporal moderada submetidas a indução/sincronização de cio.

\section{Material e Métodos}

O trabalho foi conduzido em uma fazenda particular no município de Aceguá (latitude 3149'50" Sul; longitude $\left.54^{\circ} 41^{\prime} 58 "\right)$, localizado na região fisiográfica denominada Campanha, estado do Rio Grande do Sul, no período de setembro de 2005 a abril de 2006.

Foram utilizadas 42 vacas de corte em lactação, da raça Aberdeen Angus, com média de 42 meses de idade, tamanho, peso e época de parto uniformes e condição corporal moderada, 3 em escala de 1 a 5 (Jaume \& Moraes, 2002), do parto à desmama. Os animais foram mantidos em campo natural com taxa de lotação de 0,7 unidade animal/ha (unidade animal $=450 \mathrm{~kg}$ de peso vivo).

A produção de leite foi estimada pelo método de pesagem antes e após a mamada (Beal et al., 1990; Pimentel et al., 2006). Vacas e bezerros eram separados no dia anterior ao da pesagem ( $6 \mathrm{~h}$ ) e, ao final da tarde (18 h), eram colocados a mamar para esgotamento do úbere e separados por 12 horas. Na manhã seguinte (às 6 h), eram pesados em jejum e colocados para mamar durante 20 a 30 minutos. Em seguida, foram pesados e a diferença entre os pesos foi considerada produção de leite de 12 horas, que, multiplicada por dois, serviu para estimativa da produção durante 24 horas. Foram realizadas nove pesagens, em intervalos de 21 dias, a partir da data média dos partos, totalizando um período de 189 dias. Para as pesagens, foi utilizada balança eletrônica com capacidade máxima de $1.500 \mathrm{~kg}$ e sensibilidade de $100 \mathrm{~g}$. O peso vivo e a condição corporal das vacas foram controlados do pré-parto ao desmame no momento de avaliação de cada produção de leite.

O exame ginecológico pré-acasalamento foi realizado quando as vacas apresentavam 53,6 \pm 20 dias de período pós-parto utilizando-se aparelho de ultra-sonografia (Aloka $210 \mathrm{x}$; transdutor linear de $5 \mathrm{MHz}$ ). As vacas foram classificadas (Tabela 1) em dois grupos: vacas em anestro superficial (VAS) e vacas em anestro profundo (VAP), por meio de parâmetros fisiológicos para o restabelecimento da atividade reprodutiva pós-parto em vacas de corte (Spicer \& Echternkamp, 1986; Martin et al., 1992; Wiltbank et al., 2002).

Para indução/sincronização de cio, foi utilizado o método descrito por Moraes \& Jaume (1997), empregando pessários (esponjas intra-vaginais impregnadas com $250 \mathrm{mg}$ de acetato de medroxiprogesterona) por sete dias e aplicação de $5 \mathrm{mg}$ de benzoato de estradiol (intramuscular) 
Tabela 1 - Classificação das vacas em cada condição reprodutiva pré-acasalamento

\begin{tabular}{lll}
\hline Classificação das vacas & Tônus uterino & \multicolumn{1}{c}{ Estruturas ovarianas } \\
\hline Em anestro superficial & Presença de tônus & Folículos $\geq 10 \mathrm{~mm}$ e ausência de corpo lúteo \\
Em anestro profundo & Ausência de tônus & Folículos $<10 \mathrm{~mm}$ e ausência de corpo lúteo \\
\hline
\end{tabular}

no momento da colocação e 0,5 mg no momento da retirada dos pessários. Após a retirada, os bezerros foram separados das vacas por quatro dias para inseminação artificial pelo método convencional com observação de cio. Em seqüência, as vacas foram acasaladas em monta natural por 60 dias com touro da raça Hereford. O diagnóstico de gestação foi realizado por meio de palpação trans-retal 60 dias após o término do acasalamento e as vacas controladas até o parto para verificação da data da concepção.

$\mathrm{Na}$ análise dos resultados, foram considerados fatores fixos o nível de produção de leite (NPL), considerando dois grupos de vacas, um produzindo acima (NS) e outro abaixo (NI) da média de 1.052,8 kg de leite por lactação, a gestação (GEST), a condição reprodutiva pré-acasalamento (CRPA), dividida em dois grupos (Tabela 1) e a resposta à indução/sincronização de cio (RISC), caracterizada pela resposta das vacas ao protocolo de indução/sincronização (as vacas inseminadas foram consideradas com resposta ao protocolo). Como variáveis-resposta, foram considerados os pesos das vacas no pré-parto (PPP), medido 41 dias antes da data média dos partos, ao parto ( $\mathrm{PPa})$; ao acasalamento (PA); à concepção (PC), para efeito de comparação o peso das vacas vazias, foi considerado na data média de concepção das gestantes (99 dias pósparto); e ao desmame (PD); o desempenho ponderal do parto ao desmame (GMD); e a produção de leite total num período de 189 dias (PLT). Os dados foram submetidos à análise da variância (ANOVA - GLM), no Programa NCSS 7.0 (2005), considerando o seguinte modelo:

$$
\begin{gathered}
\mathrm{Y}_{\mathrm{ijklmnop}}=\mu+\mathrm{NPL}_{\mathrm{i}}+\mathrm{GEST}_{\mathrm{j}}+\mathrm{CRPA}_{\mathrm{k}}+\mathrm{RISC}_{\mathrm{l}}+ \\
\mathrm{e}_{\mathrm{ijklmnop}}
\end{gathered}
$$

em que $Y_{\text {ijklmnop }}=$ observação de PPP, PPa, PA, PC, PD, GMD e PLT; $\mu$ = média geral; $\mathrm{NPL}_{\mathrm{i}}=$ efeito do nível de produção de leite ( $\mathrm{i}=\leq 1052,8 \mathrm{~kg},>1052,8 \mathrm{~kg}) ; \mathrm{P}_{\mathrm{j}}=$ efeito da prenhez ( $\mathrm{j}=$ prenhe, vazia); $\mathrm{CRPA}_{\mathrm{k}}=$ efeito da condição reprodutiva pré-acasalamento ( $\mathrm{k}=$ anestro profundo, anestro superficial); RISC $_{l}=$ efeito da resposta ao protocolo de indução/sincronização de cio (l= sim, não); $\mathrm{e}_{\mathrm{ijklmnop}}=$ erro experimental.

\section{Resultados e Discussão}

A condição reprodutiva pré-acasalamento indicou desempenho diferente, uma vez que as vacas em anestro superficial apresentaram maior peso pré-parto e ao parto $(\mathrm{P}<0,05)$, demonstrando que o desempenho pré-parto influencia o retorno mais precoce à atividade cíclica ovariana pós-parto e pode aumentar as chances de concepção das vacas, conforme observado por DeRouen et al. (1994) e Brauner et al. (2006). Nas demais variáveis estudadas não houve influência $(\mathrm{P}>0,05)$ da condição reprodutiva préacasalamento.

Os fatores gestação e a resposta ao protocolo de indução/sincronização de cio não influenciaram os pesos pré-parto e ao parto, provavelmente porque essa diferença não alterou um ponto de condição corporal nos dois grupos estudados, os quais permaneceram em escore moderado. A diferença de peso entre os dois grupos de vacas (Tabela 2) reduziu do acasalamento até o desmame, comprovando que as vacas em anestro profundo apresentaram ganho compensatório nesse período, promovendo equilíbrio no desempenho reprodutivo de ambos os grupos (Morrison et al.,1999; Jaume \& Moraes, 2002; Pimentel et al., 2005). Vacas em anestro superficial demonstraram tendência a desempenho reprodutivo melhor (Tabela 3), evidenciando que este grupo de vacas parece responder com maior eficiência ao protocolo de indução/sincronização de cio.

A produção de leite não influenciou as características de desempenho ponderal pré e pós-parto $(\mathrm{P}>0,05)$ e de reprodução (Tabela 3). O nível de produção de leite não teve influência sobre as variáveis de desempenho ponderal e de reprodução (Tabela 3). Os resultados obtidos confirmam que a produção de leite não influencia a condição reprodutiva nem a fertilidade pós-parto de vacas de corte com condição corporal moderada conforme observado por Pimentel et al. (2005) e Brauner et al. (2006). O equilíbrio entre as vacas de nível superior e inferior de produção de leite está relacionada ao fato de essas vacas apresentarem condição corporal moderada durante todo o período pós-parto, uma vez que a condição corporal é um fator marcante em relação ao desempenho produtivo e reprodutivo pós-parto em vacas 
Tabela 2 - Médias ajustadas e erro-padrão do peso vivo (kg) das vacas

\begin{tabular}{|c|c|c|c|c|c|c|c|c|}
\hline \multirow[t]{2}{*}{ Fator } & \multirow{2}{*}{$\begin{array}{l}\text { Número de } \\
\text { vacas }\end{array}$} & \multicolumn{5}{|c|}{ Peso } & \multirow{2}{*}{$\begin{array}{l}\text { Ganho médio } \\
\text { diário }\end{array}$} & \multirow{2}{*}{$\begin{array}{l}\text { Produção de leite } \\
\text { total }\end{array}$} \\
\hline & & Pré-parto & Ao parto & Ao acasalamento & À concepção & Ao desmame & & \\
\hline
\end{tabular}

Nível de produção de leite

\begin{tabular}{|c|c|c|c|c|c|c|c|c|}
\hline NS & 19 & $450,51 \pm 8,43$ & $379,06 \pm 8,23$ & $408,04 \pm 7,60$ & $412,19 \pm 6,82$ & $410,33 \pm 6,67$ & $0,090 \pm 0,03$ & $1.272,50 \pm 35,86^{* * *}$ \\
\hline \multicolumn{9}{|l|}{ Gestação } \\
\hline \multicolumn{9}{|l|}{ CRPA } \\
\hline $\begin{array}{l}\text { Superficial } \\
\text { Profundo }\end{array}$ & $\begin{array}{l}22 \\
20\end{array}$ & $\begin{array}{l}471,58 \pm 7,84^{*} \\
445,66 \pm 8,22^{*}\end{array}$ & $\begin{array}{l}397,50 \pm 7,65^{*} \\
374,91 \pm 8,02^{*}\end{array}$ & $\begin{array}{l}420,40 \pm 6,91 \\
403,54 \pm 7,41\end{array}$ & $\begin{array}{l}416,31 \pm 6,91 \\
403,90 \pm 7,41\end{array}$ & $\begin{array}{l}416,38 \pm 6,06 \\
408,63 \pm 6,50\end{array}$ & $\begin{array}{l}0,111 \pm 0,04 \\
0,132 \pm 0,04\end{array}$ & $\begin{array}{l}1.061,13 \pm 33,33 \\
1.044,65 \pm 34,95\end{array}$ \\
\hline \multicolumn{9}{|l|}{ RISC } \\
\hline $\begin{array}{l}\text { Não } \\
\text { Sim } \\
\text { Geral }\end{array}$ & $\begin{array}{l}25 \\
17 \\
42\end{array}$ & $\begin{array}{c}464,13 \pm 7,35 \\
453,11 \pm 8,92 \\
458,62\end{array}$ & $\begin{array}{c}392,74 \pm 7,17 \\
379,68 \pm 8,70 \\
386,21\end{array}$ & $\begin{array}{c}417,64 \pm 6,50 \\
406,29 \pm 8,04 \\
411,97\end{array}$ & $\begin{array}{c}414,62 \pm 6,50 \\
405,77 \pm 8,04 \\
411,90\end{array}$ & $\begin{array}{c}417,04 \pm 5,70 \\
407,97 \pm 7,05 \\
412,50\end{array}$ & $\begin{array}{c}0,093 \pm 0,04 \\
0,150 \pm 0,04 \\
0,122\end{array}$ & $\begin{array}{c}1.079,27 \pm 31,26 \\
1.026,51 \pm 37,91 \\
1.052,80\end{array}$ \\
\hline
\end{tabular}

de corte(Spitzer et al., 1995; Morrison et al., 1999; Wettemann et al., 2003; Hess et al., 2005).

A taxa de gestação não ocasionou diferença $(\mathrm{P}>0,05)$ nas variáveis de produção estudadas (Tabela 2). O sucesso da taxa de prenhez pode ser explicado pela tendência de maior ganho de peso destas vacas (34,73 kg) entre o parto e acasalamento em comparação às vacas vazias (16,8 kg). Esta diferença aparente indica que, em vacas de corte com condição corporal moderada do parto ao acasalamento, pequena variação de peso pré-acasalamento pode influenciar o desempenho reprodutivo. Vacas prenhes em condição corporal moderada tendem a produzir mais leite $(81,0 \mathrm{~kg})$ durante a lactação, portanto, a gestação é influenciada por outros fatores que não a produção de leite total (Pimentel et al., 2005), como provavelmente a condição reprodutiva pré-acasalamento, uma vez que $64,7 \%$ (11/17) das vacas prenhes estavam em anestro superficial (Tabela 3).

A resposta ao protocolo de indução/sincronização de cio não foi influenciada $(\mathrm{P}>0,05)$ pelas variáveis de produção estudadas (Tabela 2). As vacas que responderam ao protocolo praticamente mantiveram seus pesos durante o acasalamento e vacas que não responderam perderam peso (5 kg) no período de acasalamento (Tabela 2). Esse fato,

Tabela 3 - Análise da freqüência dos fatores estudados ${ }^{1}$

\begin{tabular}{|c|c|c|c|c|c|c|c|c|c|c|c|c|}
\hline \multirow[b]{2}{*}{ CRPA } & \multicolumn{3}{|c|}{ Condição reprodutiva pré-acasalamento } & \multicolumn{2}{|c|}{ Gestação } & \multicolumn{3}{|c|}{ Nível de produção de leite } & \multicolumn{3}{|c|}{ RISC } & \multirow[t]{2}{*}{ Total } \\
\hline & Anestro & superficial & Anestro profundo & Prenhe & Vazia & NS & & NI & & Sim & Não & \\
\hline AS & & & & $10(23,8)^{2}$ & $12(28,6)$ & $10(23,9)$ & 12 & $(28,5)$ & 10 & $(23,8)$ & $12(28,6)$ & $22(52,4)$ \\
\hline AP & & & & $6(14,3)$ & $14(33,3)$ & $9(21,4)$ & 11 & $(26,1)$ & 7 & $(16,7)$ & $13(30,9)$ & $20(47,6)$ \\
\hline \multicolumn{13}{|l|}{ Gestação } \\
\hline Prenhas & 10 & $(23,8)$ & $6(14,3)$ & & & $10(23,8)$ & 6 & $(14,3)$ & 5 & $(11,9)$ & $11(26,2)$ & $16(38,1)$ \\
\hline Vazias & 12 & $(28,5)$ & $14(33,3)$ & & & $9(21,4)$ & 17 & $(40,5)$ & 12 & $(28,6)$ & $14(33,3)$ & $26(61,9)$ \\
\hline \multicolumn{13}{|c|}{ Nível de PL } \\
\hline NS & 10 & $(23,9)$ & $9(21,4)$ & $10(23,8)$ & $9(21,4)$ & & & & 8 & $(19,1)$ & $11(26,2)$ & $19(45,2)$ \\
\hline NI & 12 & $(28,5)$ & $11(26,1)$ & $6(14,3)$ & $17(40,5)$ & & & & 9 & $(21,4)$ & $14(33,3)$ & $23(54,8)$ \\
\hline \multicolumn{13}{|l|}{ RISC } \\
\hline Sim & 10 & $(23,8)$ & $7(16,7)$ & $5(11,9)$ & $12(28,6)$ & $8(19,1)$ & 9 & $(21,4)$ & & & & $17(40,5)$ \\
\hline Não & 12 & $(28,6)$ & $13(30,9)$ & $11(26,2)$ & $14(33,3)$ & $11(26,2)$ & 14 & $(33,3)$ & & & & $25(59,5)$ \\
\hline
\end{tabular}

1 Análise realizada pelo teste de qui-quadrado.

2 Valores em porcentagem.

NS - nível superior à média; NI - nível inferior à média; RISC - resposta à indução/sincronização de cio. 
aliado à diferença individual à resposta ao protocolo de indução de cio, pode explicar a maior resposta no grupo que respondeu ao protocolo, como verificado em trabalhos anteriores (Day, 2004; Bastos et al., 2004).

\section{Conclusões}

A classificação da condição reprodutiva préacasalamento em vacas de corte pode ser um indicativo do desempenho reprodutivo durante o acasalamento em vacas de corte submetidas à indução/sincronização de cio, uma vez que vacas em anestro superficial tendem a apresentar melhor resposta à indução/sincronização de cio, porém nesse estudo essa tendência não foi detectada, abrindo assim novas perspectivas para estudos futuros. Em vacas de corte em condição corporal moderada, o desempenho ponderal pré-parto influencia a condição reprodutiva préacasalamento, aumentando a probabilidade de melhor desempenho reprodutivo.

\section{Literatura Citada}

BASTOS, G.M.; BRENNER, R.H.; WILLKE, F.W. et al. Hormonal induction of ovulation and artificial insemination in suckled beef cows under nutritional stress. Theriogenology, v.62, p.847-853, 2004.

BAUMAN, D.E.; CURRIE, B.W. Partioning of nutrients during pregnancy and lactation: A review of mechanisms involving homeostasis and homeorhesis. Journal of Dairy Science, v.63, p.1514-1529, 1980.

BEAL, W.E.; NOTTER, D.R.; AKERS, R.M. et al. Techniques for estimation of milk yield in beef cows and relationships of milk yield to calf weight gain and postpartum reproduction. Journal of Animal Science, v.68, p.937-943, 1990.

BO, G.A.; ADAMS, G.P.; CACCIA, M. et al. Ovarian follicular wave emergence after treatment with progestogen and estradiol in cattle. Animal Reproduction Science, v.39, p.193-204, 1995.

BRAUNER, C.C.; PIMENTEL, M.A.; PIMENTEL, C.A. et al. Produção de leite em bovinos de corte 11. Efeito do desempenho ponderal e produção de leite sobre a eficiência reprodutiva pósparto em vacas de corte submetidas à sincronização/indução de cio. In: REUNIÃO ANUAL DA SOCIEDADE BRASILEIRA DE ZOOTECNIA, 43., 2006, João Pessoa. Anais... João Pessoa, 2006. (CD-ROM).

BURKE, C.R.; MUSSARD, M.L.; GASSER, C.L. et al. Estradiol benzoate delays new follicular wave emergence in a dosedependent manner after ablation of the dominant ovarian follicle in cattle. Theriogenology, v.60, p.647-658, 2003.
DAY, M.L. Hormonal induction of estrous cycles in anestrous Bos taurus beef cows. Animal Reproduction Science, v.82-83, p.487-494, 2004.

DEROUEN, S.M.; FRANKE, D.E.; MORRISON, D.G. Prepartum Body condition and weight influences on reproductive performance of first-calf beef cows. Journal of Animal Science, v.72, p.1119-1125, 1994.

HESS, B.W.; LAKE, S.L.; SCHOLLJEGERDES, E.J. et al. Nutritional controls of beef cow reproduction. Journal of Animal Science, v.83, p.E90-E106, 2005.

JAUME, C.M.; MORAES, J.C.F. Importância da condição corporal na eficiência reprodutiva do rebanho de cria. Bagé: Embrapa Pecuária Sul, 2002. p.1-30. (Documentos, 43).

MARTIN, L.C.; BRFNKST, J.S.; BOURDONT, R.M. et al. Genetic effects on heifer puberty and subsequent reproduction. Journal of Animal Science, v.70, p.4006-4017, 1992.

MORAES, J.C.F.; JAUME, C.M. Sincrobovi: um pessário para sincronização de cios em bovinos. Revista Brasileira de Reprodução Animal, v.21, n.2, p.99-101, 1997.

MORRISON, D.G.; SPITZER, J.C.; PERKINS, J.L. Influence of Prepartum body condition score change on reproduction in multiparous beef cows calving in moderate body condition. Journal of Animal Science, v.77, p.1048-1054, 1999.

NCSS 7.0. Statistical System for Windows - User's Guide I, II, III. Kaysville: 2005. (CD-ROM).

PIMENTEL, M.A.; MORAES, J.C.F.; JAUME, C.M. et al. Características da lactação de vacas Hereford criadas em um sistema de produção extensivo na região da campanha do Rio Grande do Sul. Revista Brasileira de Zootecnia, v.35, n.1, p.1-11, 2006.

PIMENTEL, M.A.; MORAES, J.C.F.; JAUME, C.M. et al. Produção de leite e desempenho pós parto de vacas Hereford em distintas condições reprodutivas criadas extensivamente. Revista Ciência Rural, v.35, n.1, p.150-156, 2005.

SHORT, R.E.; BELLOWS, R.A.; STAIGMILLERET, J.G. et al. Physiological mechanisms controlling anestrus and infertility in pospartum beef cattle. Journal of Animal Science, v.68, n.3, p.799-815, 1990.

SPICER, L.J.; ECHTERNKAMP, S.E. Ovarian follicular growth, function and turnover in cattle: a review. Journal of Animal Science, v.62, p.428-451, 1986.

SPITZER, J.C.; MORRISON, D.G.; WETTEMANN, R.P. et al. Reproductive responses and calf birth and weaning weights as affected by body condition at parturition and postpartum weight gain in primiparous beef cow. Journal of Animal Science, v.73, p.1251-1257, 1995.

WETTEMANN, R.P.; LENTS, C.A.; CICCIOLI, N.H. et al. Nutritional- and suckling-mediated anovulation in beef cows. Journal of Animal Science, v.81, p.E48-E59, 2003.

WILLIAMS, G.L. Suckling as a regulator of postpartum rebreeding in cattle: a review. Journal of Animal Science, v.68, p.831852, 1990.

WILTBANK, M.C.; GTIMEN, A.; SARTORI, R. Physiological classification of anovulatory conditions in cattle. Theriogenology, v.57, p.21-52, 2002.

YAVAS, Y.; WALTON, J.S. Induction of ovulation in postpartum suckled beef cows: a review. Theriogenology, v.54, p.1-23, 2000 . 